\title{
Early experience of laparoscopic liver resection: A single institution experience with 37 consecutive cases
}

\author{
Eun Jeong Jang and Kwan Woo Kim
}

Department of Surgery, Dong-A University College of Medicine, Dong-A University Medical Center, Busan, Korea

\begin{abstract}
Backgrounds/Aims: Laparoscopic liver resection (LLR) has evolved and broadened in scope. While open liver resections are currently being performed safely in our hospital, LLRs are being implemented in fewer cases. The aim of this study was to review our initial experience in LLR to assess early outcomes of the procedure. Methods: A retrospective chart review was conducted for 37 patients who underwent laparoscopic liver resections for various indications between January 2014 and July 2017 by a single surgeon who had performed 161 open liver resections and 50 live donor hepatectomies during the same period. Results: Of 37 laparoscopic liver resections performed, male to female ratio was 23 to 4 . Their mean age was 61.4 years. There were 13 cases of wedge resections, 7 cases of left lateral sectionectomy, 9 cases of left hepatectomy, and 8 cases of right hepatectomy. Pathology included hepatocellular carcinoma $(n=20)$, cholangiocarcinoma $(n=3)$, intrahepatic duct stones $(n=6)$, metastatic liver carcinoma $(n=6)$, primary neuroendocrine tumor of liver $(n=1)$, and huge hemangioma $(n=1)$. The mean operation time was 174.7 minutes (range, 40-410 minutes). Mean blood loss was $200.5 \mathrm{ml}$ (range, 10-2200 ml). There were no open-conversion cases. There were no intraoperative or postoperative complications except that a case of severe portal vein stenosis in the laparoscopic right hepatectomy occurred postoperatively. The patient underwent reoperation (portal vein resection and anastomosis, stenting). The mean hospital stay was 8.7 days (range, 2-44 days). Conclusions: Even though our experience in laparoscopic liver resection is still developing, our results are comparable to those of other studies. Therefore, an experienced surgeon in performing open liver resection should be able to perform the laparoscopic liver resection safely. (Ann Hepatobiliary Pancreat Surg 2019;23:115-121)
\end{abstract}

Key Words: Laparoscopic liver resection; Laparoscopic major hepatectomy; Open liver resection

\section{INTRODUCTION}

Laparoscopic liver resection (LLR) was first introduced in the early 1990s. ${ }^{1}$ Since then, it has been rapidly accepted as a safe and feasible option for the treatment of various benign and malignant liver lesions. However, the adoption of LLR by liver surgeons has been relatively slow due to the technical complexity of LLR, and many liver surgeons remain reluctant to perform it even today. Nevertheless, improvements in biomedical technology and the accumulation of surgical experience have led to a global increase in the number of LLRs performed. ${ }^{2-9}$

The evolution of LLR has led to the 2008 international consensus that LLRs are eventually acknowledged as safe procedures with acceptable morbidity and mortality for both minor and major liver resections when performed by certified hepatobiliary surgeons with experience in laparoscopic surgery. ${ }^{10}$

Despite the clear feasibility and safety of laparoscopic minor hepatectomy in many studies, ${ }^{2-5}$ laparoscopic major resection (LMR) remains challenging due to technical difficulties and fear of uncontrolled bleeding. Therefore, laparoscopic major hepatectomy has been performed mainly in large centers.

Although our center does not have a large volume, we have $>10$ years of accumulated experience with open liver surgery and laparoscopic surgery for various liver resections and living donor liver transplantations with a high success rate.

The objective of this study was to present our early ex-

Received: October 7, 2018; Revised: December 14, 2018; Accepted: December 20, 2018

Comesponding author: Kwan Woo Kim

Division of Liver Transplantation and Hepato-Biliary-Pancrease Surgery, Department of Surgery, Dong-A University College of Medicine, Dong-A University Medical Center, 26 Daesingongwon-ro, Seo-gu, Busan 49201, Korea

Tel: +82-51-240-5146, Fax: +82-51-240-9316, E-mail: d002045@gmail.com

Copyright (C) 2019 by The Korean Association of Hepato-Biliary-Pancreatic Surgery

This is an Open Access article distributed under the terms of the Creative Commons Attribution Non-Commercial License (http://creativecommons.org/ licenses/by-nc/4.0) which permits unrestricted non-commercial use, distribution, and reproduction in any medium, provided the original work is properly cited. Annals of Hepato-Biliary-Pancreatic Surgery - pISSN: 2508-5778 - elSSN: 2508-5859 
perience in laparoscopic minor and major hepatectomy and assess early outcomes of the procedure by one certified hepatobiliary surgeon based on our open surgery experience.

\section{MATERIALS AND METHODS}

A retrospective chart review was conducted of 37 patients who underwent pure laparoscopic liver resections for various indications between January 2014 and July 2017 by a single surgeon, who had performed 161 open liver resections ( $>3$ segments/ $<3$ segments: 120/41) and 50 live donor hepatectomies during the same period in (Table 1).

Additionally, the surgeon had experience with various laparoscopic surgery types (laparoscopic cholecystectomy, distal pancreatectomy with or without splenectomy and adrenalectomy) for approximately 1500 cases.

The study was approved by the ethics committee of Dong-A University College of Medicine, Dong-A University Medical Center of Korea (IRB number: DAUHIRB18-100). Informed consent was obtained from all patients.

Laparoscopic major resection was defined as the resection of 3 liver segments or more.

All patients had good preoperative performance status (American Society of Anesthesiology classes I to III). Routine blood tests, measurement of viral titers, liver dynamic computed tomography $(\mathrm{CT})$, or magnetic resonance imaging (MRI) were performed in all patients. Additionally, positron emission tomography (PET) scans, chest CT

Table 1. Type of resection for patients undergoing open liver resection for various indications

\begin{tabular}{lc}
\hline \multicolumn{1}{c}{ Operation name } & No. \\
\hline Wedge resection of the liver & 11 \\
Left lateral sectionectomy & 10 \\
Left hepatectomy & 35 \\
Extended left hepatectomy & 9 \\
Right anterior sectionectomy & 8 \\
Right posterior sectionectomy & 9 \\
Extended right posterior sectionectomy & 3 \\
Central bisectionectomy & 6 \\
Right hepatectomy & 60 \\
Left trisectionectomy & 3 \\
Right trisectionectomy & 7 \\
Donor hepatectectomy & 50 \\
Total & 211 \\
\hline
\end{tabular}

scans, and tumor markers were assessed in patients who had malignant liver diseases. When considering laparoscopic major liver resection, we checked the expected remnant liver volume for all patients by CT liver volumetry. The cut-off value was $35 \%$ of total liver volume for all patients. Tumors that were bilateral, large $(>10 \mathrm{~cm})$, involved major hepatic or portal veins, or were located within $1 \mathrm{~cm}$ of the hepatic hilum or inferior vena cava (IVC) were excluded for laparoscopic major hepatectomy. This exclusion criteria are very similar to those of the Asan Medical Center, Seoul, Korea. ${ }^{11}$

\section{Surgical technique}

We do not describe here the method of wedge resection and left lateral sectionectomy because the procedures are simple. We only refer to the laparoscopic major liver resection.

This surgical technique is also very similar to that of Asan Medical Center, Seoul, Korea. ${ }^{11}$

The patient was placed in the supine and mild reverse Trendelenburg position, with the legs spread apart (the French position). The operating surgeon stood between the patient's legs. The camera and the assistant surgeon were positioned on the patient's left. The intermittent pneumatic compressor was used on the legs of all patients who underwent laparoscopic major hepatectomy to prevent deep vein thrombosis. Five trocars were used in all procedures: four $12 \mathrm{~mm}$ ports and one $5 \mathrm{~mm}$ port. Trocar position was determined by the type of operation and the location of the tumor. We used a 10-mm, 30-degree camera. Pneumoperitoneum was established with a Veress needle, and the intra-abdominal pressure was maintained between 12-14 mmHg with $\mathrm{CO}_{2}$ gas.

After dividing the round ligament, dissection of the falciform ligament was performed in a cephalad direction and the root of the hepatic vein was exposed. For a right hepatectomy, inflow was controlled by the Glissonean approach. After cholecystectomy, the peritoneum of the hepatoduodenal ligament was dissected at the hilar region. The glissonean pedicle was encircled extraparenchymally using a Goldfinger dissector (Ethicon Endo Surgery, Johnson \& Johnson, New Brunswick, NJ, USA). The isolated Glissonean pedicle was clamped with a laparoscopic bulldog clamp, and the demarcation line was identified and marked with electrocautery. For left hemihepatectomy, 
cholecystectomy was not performed for traction during parenchymal transection unless there were no pathologic findings concerning the gallbladder. To control the inflow in the same way as used in the right Glissonean approach, the left Glissonean pedicle was encircled extraparenchymally using the Goldfinger dissector. The isolated Glissonean pedicle was clamped with a laparoscopic bulldog clamp; then, the demarcation line was identified and marked with electrocautery.

We used the Pringle maneuver to prevent bleeding during the parenchymal transection. Superficial parenchyma of the liver was transected using energy devices including the Harmonic Scalpel (Ethicon Endo-Surgery Inc., Cincinnati, OH, USA). Deep parenchymal transection was performed with a combination of Cavitron Ultrasonic Surgical Aspirator (CUSA; Excel, Integra Lifesciences Co., Plainsboro, NJ, USA) and the energy devices. Small branches of the hepatic vein and Glisson' sheath were controlled with endoclips. The hepatic vein and Glissonean pedicle were transected using vascular staplers.

The resected specimen was retrieved using a plastic bag via a suprapubic incision at the right hepatectomy. On the other hand, when we performed a left hepatectomy, the umbilical wound was extended, and then the resected specimen was retrieved. After hemostasis and irrigation of the surgical bed, one closed-suction drainage tube was

Table 2. Demographic data and pathologic results of patients undergoing laparoscopic liver resection

\begin{tabular}{lrl}
\hline \multicolumn{1}{c}{ Demographics } & \multicolumn{1}{c}{ No./range } \\
\hline Mean age, y, range & $61.4 \pm 10.0(42-79)$ \\
Sex, male/female (\%) & $23 / 14(62.2 / 37.8)$ \\
Mean Body Mass Index, kg/m ${ }^{2}$ & $23.6 \pm 2.6$ & $(19.3-30.7)$ \\
ASA status, n (\%) & $4(10.8)$ \\
I & $24(64.8)$ \\
II & $9(24.3)$ \\
III & $20(54.1)$ \\
Pathologic diagnosis & $6(16.2)$ \\
Hepatocellular carcinoma, n (\%) & $6(16.2)$ \\
Metastatic carcinoma, n (\%) & $3(8.1)$ \\
Hepatolithiasis, n (\%) & $1(2.7)$ \\
Cholangiocarcinoma, n (\%) & $1(2.7)$ \\
Neuroendocrine tumor of liver, n (\%) & \\
Huge hemangioma, n (\%) & $2.7 \pm 2.0(1-9)$ \\
Pathologic result & $14.8 \pm 15.7(1-45)$ \\
Mean tumor size, cm & \\
Mean resection margin, mm
\end{tabular}

ASA, American Society of Anesthesiology routinely placed near the surgical bed.

\section{Statistical analysis}

All statistical analyses were conducted using the Statistical Package for Social Sciences for Windows, version 21.0 (SPSS Inc, Chicago, IL, USA). Categorical variables were summarized using proportions, and continuous variables were presented as the mean with standard deviation.

\section{RESULTS}

A total of 37 patients (23 males, 14 females) underwent laparoscopic liver resection, including 13 wedge resections of liver, 7 left lateral sectionectomies, 8 left hepatectomies, 1 left hepatectomy+S5 segmentectomy, 8 right hepatectomies in (Tables 1 and 2).

Of these, 17 cases (49.5\%) were laparoscopic major hepatectomies. The mean operating time was $174.7 \pm 104.2$ min. The estimated blood loss was $200.5 \pm 436.3 \mathrm{ml}$, and intraoperative blood transfusion was required in 7 (18.9\%) patients. The mean hospital stay was $8.7 \pm 6.7$ days. There was no open conversion in (Table 2).

The mean age of the patients was $61.4 \pm 10.0$ years, and their mean body mass index was $23.6 \pm 2.6 \mathrm{~kg} / \mathrm{m}^{2}$. American Society of Anesthesiology classes I, II, and III patients accounted for 4 (10.8\%), 24 (64.8\%) and 9 (24.3\%) individuals in (Table 3 ), respectively.

Pathologic date is summarized in (Table 3). Pathologic

Table 3. Surgical outcomes and type of resection for patients undergoing laparoscopic liver resection

\begin{tabular}{lr}
\hline \multicolumn{1}{c}{ Outcomes } & No./range \\
\hline Type of resection & \\
Wedge resection of the liver, n (\%) & $73(35.1)$ \\
Left lateral sectionectomy, n (\%) & $8(21.6)$ \\
Left hepatectomy, n (\%) & $1(2.7)$ \\
Left hepatectomy+ & $8(21.6)$ \\
S5 segmentectomy, n (\%) & $17 / 20(45.9 / 54.1)$ \\
Right hepatectomy, n (\%) & $7(18.9)$ \\
Major/minor resection, n (\%) & \\
Mean operation time, min (range) & $174.7 \pm 104.2(40-410)$ \\
Mean estimated blood loss, & $200.5 \pm 436.3(0-2200)$ \\
ml (range) & \\
Intraoperative blood transfusion, & \\
$\mathrm{n}(\%)$ & $0.7 \pm 6.7(2-44)$ \\
Length of hospital stay, days & $(0)$ \\
Open conversion, n (\%) &
\end{tabular}


diagnoses were $20(54.1 \%)$ hepatocellular carcinoma, 6 (16.2\%) metastatic carcinoma, $6(16.2 \%)$ hepatolithiasis, $3(8.1 \%)$ cholangiocarcinoma, $1(2.7 \%)$ neuroendocrine tumor of liver and $1(2.7 \%)$ huge hemangioma. The mean tumor size and mean resection margin was $2.7 \pm 2.0 \mathrm{~cm}$ and $14.8 \pm 15.7 \mathrm{~mm}$, respectively.

According to the Clavien-Dindo classification, 1 complication was grade I; fluid collection at the liver cut surface was resolved by conservative management in (Table 4).

One patient had a complication, C-D classification (grade III-B), of severe portal vein stenosis after laparoscopic right hepatectomy. We performed a second operation (portal vein resection and anastomosis using cryopreserved iliac vein).

Pre- and postoperative data for undergoing laparoscopic major hepatectomies are summarized in (Table 5).

The mean operating time was $252.6 \pm 94.5 \mathrm{~min}$. The estimated blood loss was $265.2 \pm 514.9 \mathrm{ml}$, and intraoperative blood transfusion was required in $7(41.2 \%)$ patients. The mean hospital stay was $11.6 \pm 8.7$ days. There was no open conversion. Pathologic diagnoses were 7 (41.2\%) hepatocellular carcinoma, 5 (29.4\%) hepatolithiasis, 3 (17.6\%) cholangiocarcinoma, 1 (5.9\%) metastatic carcinoma, and $1(5.9 \%)$ huge hemangioma.

The mean tumor size and mean resection margin were $3.7 \pm 2.9 \mathrm{~cm}$ and $23.8 \pm 18.5 \mathrm{~mm}$, respectively.

According to the Clavien-Dindo classification, as already mentioned, 1 complication was grade I, and the other was grade III-B (severe stenosis and thrombosis of the portal vein after laparoscopic right hepatectomy). There was no mortality within the first postoperative 90 days. Additionally, there was no open conversion.

Table 4. Mortality and morbidity for patients undergoing laparoscopic liver resection

\begin{tabular}{lc}
\hline \multicolumn{1}{c}{ Mortality and morbidity } & No. \\
\hline Mortality, 90 day, n (\%) & $0(0.0)$ \\
Morbidity, n (\%) & $2(5.4)$ \\
Clavien-Dindo I, n (\%) & $1(2.7)$ \\
Fluid collection at liver cut surface & $1(2.7)$ \\
Clavien-Dindo III-B, n (\%) & \\
Reoperation (portal vein stenosis) & \\
\hline
\end{tabular}

\section{DISCUSSION}

Laparoscopic liver surgery has undergone major evolution during the past decade. Although initially approached with restraint and reluctance by the surgical community, LLR is currently considered a safe and adequate approach in the management of liver lesions in the hands of experienced surgeons. ${ }^{11}$

In 2007, a series of 300 minimally invasive hepatectomies were reported by Koffron et al. ${ }^{3}$ The increasingly encouraging outcomes in multiple series led to the 2008 international consensus (Louisville. Kentucky, USA), where, following thorough evaluation, LLRs were recognized as safe and efficient procedures when performed by proficient hepatobiliary surgeons. ${ }^{10}$

Laparoscopic major hepatectomy (LMH) was first reported in 1998, and its development has been relatively slow. ${ }^{3}$ The first series of LMH procedures were published in $2004,{ }^{12}$ and subsequently, only a few other series were published over the next few years. 13,14

Significant risk of uncontrollable hemorrhage is the

Table 5. Pre- and postoperative data for patients undergoing laparoscopic major hepatectomy

\begin{tabular}{lrl}
\hline \multicolumn{1}{c}{ Outcomes } & \multicolumn{1}{c}{ No./range } \\
\hline Mean age, y, range & $61.9 \pm 9.1(43-79)$ \\
Sex, male/female (\%) & $10 / 7(58.8 / 41.2)$ \\
Mean Body Mass Index, kg/m² & $23.8 \pm 3.0(19.5-30.7)$ \\
Pathologic diagnosis & $7(41.2)$ \\
Hepatocellular carcinoma, n (\%) & $5(29.4)$ \\
Hepatolithiasis, n (\%) & $3(17.6)$ \\
Cholangiocarcinoma, n (\%) & $1(5.9)$ \\
Metastatic carcinoma, n (\%) & $1(5.9)$ \\
Huge hemangioma, n (\%) & \\
Pathologic result & $3.7 \pm 2.9(1-9)$ \\
Mean tumor size, cm & $23.8 \pm 18.5(1-45)$ \\
Mean resection margin, mm & \\
Mean operation time, min (range) & $252.6 \pm 94.5(100-410)$ \\
Mean estimated blood loss, ml, range & $265.2 \pm 514.9(10-2200)$ \\
Intraoperative blood transfusion, & $7(41.2)$ \\
$\mathrm{n}$ (\%) & \\
Length of hospital stay, days & $11.6 \pm 8.7(8-44)$ \\
Open conversion, n (\%) & $0(0)$ \\
Mortality, 90 day, n (\%) & $0(0.0)$ \\
Morbidity, n (\%) & $2(25)$ \\
Fluid collection at liver cut sur- & $1(12.5)$ \\
face, n (\%) & $1(12.5)$ \\
Reoperation (portal vein stenosis), & \\
n (\%) & \\
\hline
\end{tabular}


main concern when attempting LMH. Early series of LMH reported by pioneering surgeons and early adopters demonstrated the steep learning curves associated with LMH. ${ }^{15}$ In their first experience of 23 consecutive laparoscopic right hepatectomies at Henri Mondor Hospital, France, six $(26 \%)$ cases were converted to the open procedure, including two of the first three cases, despite the investigators having significant experience with laparoscopic minor hepatectomies. ${ }^{15}$

Hence, due to the technical complexities of total LMH and its steep learning curve, hand-assistance ${ }^{12,16}$ and hybrid procedures (laparoscopic assistance) ${ }^{17}$ have been proposed by several authors, especially during the initial learning phase. In a review published in 2009, of 210 LMH performed at six experienced international centers, pure laparoscopy was only performed in $43 \%$ of cases, whereas $57 \%$ were performed via hand-assistance. ${ }^{18}$ The open-conversion rate was $12 \%$. In this review, some centers utilized hand-assistance or the hybrid procedure selectively for $\mathrm{LMH}$, especially during the initial learning experience.

Systematic review documented complications in $11 \%$ to $23 \%$ of patients, with 3 deaths among 770 patients. $^{1-3}$ In this study, there were 37 LLR for both minor and major hepatectomies, and 17 (45.9\%) of 37 cases were LMHs. Unlike other studies, a big portion of the cases were LMH. There was no mortality within the first postoperative 90 days and no open conversion. The morbidity rate was $5.4 \%$. Considering that we have been performing LMH only since 2015, our 3-year results are outstanding. As seen in Table 3, the surgical results of laparoscopic minor hepatectomy are very good, and the surgical results of LMH are comparable to other large volume centers. ${ }^{11}$ Though the number of cases is small, there was no open conversion or use of hand-assistance or the hybrid procedure during our initial learning experience.

Only one patient, who was a 58-year-old male with multiple HCCs on S5 and S7, had a major complication and needed reoperation. He underwent a laparoscopic right hepatectomy; after the operation, liver function was deteriorated, and large amount of ascites came out. At postoperative day 6 , we checked the CT scan and diagnosed severe portal vein stenosis and thrombosis. Then, at postoperative day 9, we performed a second operation (portal vein resection and anastomosis using cryopreser- ved iliac vein).

The patient had a type III portal vein in which 3 branches are divided at the same point. In the laparoscopic right hepatectomy procedure, we used a linear stapling device to ligate and resect the Glissonean pedicle. We found that the 1st staple was properly applied at the right anterior Glissonean pedicle but that the 2nd staple for the right posterior Glissonean pedicle had a placement error, and severe main portal vein narrowing had occurred. After the reoperation, although there was no specific problem with liver function, we still noted mild stenosis of the portal vein, which was confirmed on CT scan. Therefore, we performed portal vein stenting as an intervention at postoperative day 20. The intervention was successful, and the patient was discharged at postoperative day 44. As of now, the patient is doing well, without recurrence, and has regularly visited the outpatient clinic for approximately 3 years.

When we refer to our results, we have two important points to mention.

First, although we have experience only with early laparoscopic liver resection, we were able to perform LMH without long learning curves through many open hepatectomies, various laparoscopic experiences, and liver transplants.

In addition, we have been able to perform pure laparoscopic hepatic resection without the hybrid method used in the early stage of presentation at many centers. We believe that our lower morbidity rates in this study are mainly due to strict patient selection, precise anatomical resection, meticulous operative techniques, and a wealth of experience with open major hepatectomy.

Second, based on our case with complications, we think the ability to overcome major complications that could arise during LMH is also important. Many open hepatectomy experiences could require the ability to cope with complications that may occur during or after laparoscopic surgery. This open hepatectomy experience has given us the confidence to perform well in laparoscopic major hepatectomy.

As mentioned earlier, when doing the laparoscopic right hepatectomy, we did a pure anterior approach. The isolated right Glissonean pedicle was clamped with a laparoscopic bulldog clamp; then, the demarcation line was identified and marked with electrocautery. We performed 
liver transection with CUSA immediately. The patient underwent surgery in the order of Glissionean pedicle transection, right hepatic vein resection, right lobe mobilization, and specimen removal.

This procedure is possible because there is a good laparoscopic instrument, the Goldfinger dissector. We have applied the Goldfinger dissector to many different laparoscopic surgeries, and based on this experience, we feel we could perform well on pure LMH. The atraumatic tip and multi-positional flexibility are ideal for safe isolation of the Glissonean pedicle and right hepatic vein.

The extraparenchymal Glissonean approach was preferred for the right-side hepatectomy, and individual dissection was preferred for the left hepatectomy for inflow control. In the left hepatectomy, individual isolation and division of the hepatic artery and portal vein was much easier and more convenient than it was when using the Glissonean approach. ${ }^{11}$

When performing a left hepatectomy, many centers prefer individual dissection, but we use an extraparenchymal Glissionean approach, based on our experience of open left liver resection. Our procedure for laparoscopic left hepatectomy is very useful and shortens the operation time, unlike results from other studies. If we have more experience with the left hepatectomy, we will introduce extraparenchymal Glissionean approach for control inflow during laparoscopic left hepatectomy.

The oncologic outcome after LMH is a major issue, and long-term survival data on patients who underwent laparoscopic major liver resection for HCC are lacking. However, several studies have reported that the overall survival rate and disease-free survival rate of laparoscopic liver resection patients are similar to those of individuals undergoing open liver surgery during short-term followup. $^{19-21}$

In this study, 20 of 37 cases were HCCs. Although oncological outcome is not mentioned here, if more cases accumulate in the future, we think it is necessary to study not only the surgical outcome but also the oncological outcome.

We discussed the surgical results of our initial small experience in laparoscopic liver resection. Although the number of cases is small, results are comparable with other studies, in terms of operating time, estimated blood loss, length of hospital stay, morbidity, mortality, and open-conversion rate.

Finally, it is important to emphasize the Louisville Consensus in 2008: laparoscopic hepatectomy, especially major hepatectomies, should only be attempted by surgeons with extensive experience and expertise in both open hepatectomy and laparoscopic surgery.

Although our experience in laparoscopic liver resection is not as extensive as others, our results are comparable to those in other studies. Therefore, an experienced surgeon in open liver resection should be able to perform laparoscopic liver resection without a long learning curve.

Especially from the standpoint of laparoscopic major hepatectomy, great experience in both open liver and laparoscopic surgery is needed to perform laparoscopic major liver resection safely.

\section{REFERENCES}

1. Gagner M, Rheault M, Dubuc J. Laparoscopic partial hepatectomy for liver tumor [abstract]. Surg Endosc 1992;6:99.

2. Vibert E, Perniceni T, Levard H, Denet C, Shahri NK, Gayet B. Laparoscopic liver resection. Br J Surg 2006;93:67-72.

3. Koffron AJ, Auffenberg G, Kung R, Abecassis M. Evaluation of 300 minimally invasive liver resections at a single institution: less is more. Ann Surg 2007;246:385-392; discussion 392-394.

4. Dagher I, Proske JM, Carloni A, Richa H, Tranchart H, Franco D. Laparoscopic liver resection: results for 70 patients. Surg Endosc 2007;21:619-624.

5. Nguyen KT, Gamblin TC, Geller DA. World review of laparoscopic liver resection-2,804 patients. Ann Surg 2009;250:831841.

6. Croome KP, Yamashita MH. Laparoscopic vs open hepatic resection for benign and malignant tumors: an updated meta-analysis. Arch Surg 2010;145:1109-1118.

7. Mirnezami R, Mirnezami AH, Chandrakumaran K, Abu Hilal M, Pearce NW, Primrose JN, et al. Short- and long-term outcomes after laparoscopic and open hepatic resection: systematic review and meta-analysis. HPB (Oxford) 2011;13:295-308.

8. Reddy SK, Tsung A, Geller DA. Laparoscopic liver resection. World J Surg 2011;35:1478-1486.

9. Xiong JJ, Altaf K, Javed MA, Huang W, Mukherjee R, Mai G, et al. Meta-analysis of laparoscopic vs open liver resection for hepatocellular carcinoma. World J Gastroenterol 2012;18:66576668.

10. Buell JF, Cherqui D, Geller DA, O'Rourke N, Iannitti D, Dagher I, et al. The international position on laparoscopic liver surgery: the Louisville Statement, 2008. Ann Surg 2009;250:825-830.

11. Kang SH, Kim KH, Shin MH, Yoon YI, Kim WJ, Jung DH, et al. Surgical outcomes following laparoscopic major hepatectomy for various liver diseases. Medicine (Baltimore) 2016; 95:e5182.

12. Kluger MD, Vigano L, Barroso R, Cherqui D. The learning curve in laparoscopic major liver resection. J Hepatobiliary Pancreat Sci 2013;20:131-136.

13. O'Rourke N, Fielding G. Laparoscopic right hepatectomy: surgical technique. J Gastrointest Surg 2004;8:213-216.

14. Gayet B, Cavaliere D, Vibert E, Perniceni T, Levard H, Denet 
C, et al. Totally laparoscopic right hepatectomy. Am J Surg 2007;194:685-689.

15. Dagher I, Caillard C, Proske JM, Carloni A, Lainas P, Franco D. Laparoscopic right hepatectomy: original technique and results. J Am Coll Surg 2008;206:756-760.

16. Buell JF, Thomas MT, Rudich S, Marvin M, Nagubandi R, Ravindra KV, et al. Experience with more than 500 minimally invasive hepatic procedures. Ann Surg 2008;248:475-486.

17. Cardinal JS, Reddy SK, Tsung A, Marsh JW, Geller DA. Laparoscopic major hepatectomy: pure laparoscopic approach versus hand-assisted technique. J Hepatobiliary Pancreat Sci 2013;20: 114-119.

18. Lin NC, Nitta H, Wakabayashi G. Laparoscopic major hep- atectomy: a systematic literature review and comparison of 3 techniques. Ann Surg 2013;257:205-213.

19. Dagher I, O'Rourke N, Geller DA, Cherqui D, Belli G, Gamblin $\mathrm{TC}$, et al. Laparoscopic major hepatectomy: an evolution in standard of care. Ann Surg 2009;250:856-860.

20. Lesurtel M, Cherqui D, Laurent A, Tayar C, Fagniez PL. Laparoscopic versus open left lateral hepatic lobectomy: a case-control study. J Am Coll Surg 2003;196:236-242.

21. Yin Z, Fan X, Ye H, Yin D, Wang J. Short- and long-term outcomes after laparoscopic and open hepatectomy for hepatocellular carcinoma: a global systematic review and meta-analysis. Ann Surg Oncol 2013;20:1203-1215. 